\title{
The Influence of Reduction Conditions on a Ni-YSZ SOFC Anode Microstructure and Evolution
}

\author{
H. Monzón ${ }^{\mathrm{a}}$, M.A. Laguna-Bercero ${ }^{\mathrm{a}}$ \\ ${ }^{a}$ Instituto de Ciencia de Materiales de Aragón, Universidad de Zaragoza - CSIC, \\ Zaragoza, Spain
}

\begin{abstract}
Ni-YSZ cermets are the most widespread material used as SOFC anodes. These materials are generally fabricated through the reduction of a NiO-YSZ composite, but the reduction conditions have a great effect in the final microstructure of the electrode. In the present work several conditions were explored to reduce microtubular anode supports produced via extrusion in order to find a suitable microstructure for SOFC anodes. Samples were reduced in pure and diluted $\mathrm{H}_{2}$, either dry or humidified at temperatures ranging from 400 to $800^{\circ} \mathrm{C}$ while their DC conductivity was being monitored. The highest value of peak conductivity was measured in the sample reduced in humidified pure hydrogen at $800^{\circ} \mathrm{C}$, but it experienced more severe conductivity degradation that other samples. The best value for the duration of the test was obtained for the sample reduced in dry diluted hydrogen at $800^{\circ} \mathrm{C}$.
\end{abstract}

\section{Introduction:}

The most extended composition for solid oxide fuel cell (SOFC) anodes consists on a cermet formed by nickel, which acts as a catalyst for hydrogen dissociation and current collector, and YSZ (yttria stabilized zirconia), which acts as ionic conductor. Although some fabrication techniques may start from metallic nickel or nickel salts, most of them use nickel oxide as a precursor for the formation of the nickel phase, relying on the first exposure of the electrode to the fuel stream (reducing atmosphere) to conform the final electrode composition. When the used fuel is hydrogen, NiO reacts to form metallic nickel as shown in eq. 1 . This reaction features a volume shrinkage close to $40 \%$, forming a pore network through the volume previously filled by the NiO phase. As the actual reaction taking place during the cell operation occurs in the $1 \mathrm{D}$ region known as the triple phase boundary (TPB, YSZ-Nickel-pore interphase), the microstructure resulting after the $\mathrm{NiO}$ reduction reaction is key towards the electrode performance, being the maximization of the length of this region desirable. Additionally, connection across the nickel phase is also critical as isolated Ni particles, though able of fuel dissociation on their surface, will not contribute towards fuel oxidation during the cell operation. The final Ni-YSZ microstructure is largely determined by the initial NiOYSZ material used, here particle sizes and percolation thresholds should be considered. On the present work an exploration was performed on how NiO reducing conditions affect the final Ni-YSZ microstructure on a given tubular anode substrate. Although literature can be found on the present subject, there is no general agreement on how this parameters affect the final sample properties, and an experimental characterization is the most reliable procedure of elucidating how the reduction process affects each given microstructure. In the present work we will study standard anode supports produced via extrusion as described in (1), composition details and phase portions are given in table I. 
TABLE I. General properties of the initial material used in the tests.

\begin{tabular}{|c|c|c|}
\hline $\begin{array}{c}\text { Anode } \\
\text { composition }\end{array}$ & $\begin{array}{c}\text { Phase } \\
\text { [vol\%] }\end{array}$ & $\begin{array}{c}\text { Characteristic } \\
\text { particle size }[\boldsymbol{\mu m}]\end{array}$ \\
\hline Nickel & $29 \%$ & $0.2-3$ \\
\hline YSZ & $28 \%$ & $0.2-1$ \\
\hline Pore & $43 \%$ & $0.1-10$ \\
\hline \multicolumn{2}{|c|}{ Tubular sample parameters } \\
\hline Length $[\mathrm{cm}]$ & 5 \\
\hline$D_{\text {in }}[\mathrm{cm}]$ & 0.2 \\
\hline$D_{\text {out }}[\mathrm{cm}]$ & \multicolumn{2}{|c}{0.32} \\
\hline
\end{tabular}

There is general agreement in the literature $(2,3)$, that the mechanism for reaction in eq. 1 consists on an initial induction (i.e. nucleation) phase that takes place in the surface of $\mathrm{NiO}$ particles followed by reaction in the interface of the newly formed nickel and the nickel oxide. This second phase proceeds autocatalyticaly (metallic nickel acts as catalyst for the hydrogen dissociation), and thereby this second step shows faster kinetics.

$$
\mathrm{NiO}+\mathrm{H}_{2} \rightarrow \mathrm{Ni}+\mathrm{H}_{2} \mathrm{O}
$$

On the other hand, nickel shows fast coarsening at common SOFC temperatures, due to high diffusive movility. This coarsening is driven by the minimization of surface energy, and is considered an issue regarding long term operation due to the loss of TPB length and inter-particle connectivity. On the other hand, water effect on this mechanism is not yet fully understood. There is evidence confirming an increase in $\mathrm{Ni}$ movility due to the formation of a $\mathrm{Ni}_{2}-\mathrm{OH}$ dimer complex (4). Additionally water presence in the gas feed stream has been observed to cause an increase in the initial induction phase during reduction (3).

\section{Experimental procedure:}

Five anode support samples fabricated via extrusion and sintered at $1500^{\circ} \mathrm{C}$ were reduced in different conditions. Reduction conditions are summarized in table II. During the reduction a four point probe potenciostatic test was performed at $20 \mathrm{mV}$, allowing an indirect measure of the evolution of the reduction reaction via the DC conductivity of the sample, similar to the one described in (5). The limitation of this method is that it is only useful once an initial percolating $\mathrm{Ni}$ phase has been established across the sample. This test was performed using a Princeton Applied Research VSP potentiostat/galvanostat. Samples were electrically contacted with gold wire and gold paint was added to improve the contact. Samples were then introduced inside an alumina tube which was later sealed on both edges to rubber tubing in order to introduce the desired atmosphere. The alumina sample container was then placed inside a home made small tubular furnace with a homogeneous hot zone of $5 \mathrm{~cm}$ in length. Each sample was heated to the temperature selected for each test while fed with a $\mathrm{N}_{2}$ flow of $200 \mathrm{ml} \mathrm{min}^{-1}$. Once the selected temperature was reached, the gas feed flow was switched to the reducing gas specified in table II for each test. All tests were performed without an additional water addition in the gas feed except for sample named HTW, for 
this sample the feed stream was passed through a bubbler at room temperature. Each sample was kept in reducing conditions for 20 hours. Some of the samples experienced an electrical contact failure during the test, and no data was registered after that.

TABLE II. Test conditions for the reduction experiments.

\begin{tabular}{|c|c|c|c|c|}
\hline Sample ID & T [ $\left.{ }^{\circ} \mathbf{C}\right]$ & $\begin{array}{c}\mathbf{H}_{2} \text { flow } \\
{\left[\mathbf{c m}^{\mathbf{3}} \mathbf{m i n}^{-\mathbf{1}}\right]}\end{array}$ & $\begin{array}{c}\mathbf{N}_{\mathbf{2}} \text { flow } \\
{\left[\mathbf{c m}^{\mathbf{3}} \mathbf{m i n}^{-\mathbf{1}} \mathbf{]}\right.}\end{array}$ & $\begin{array}{c}\text { Humidified } \\
\mathbf{( R T )}\end{array}$ \\
\hline HTD & 800 & 90 & 0 & NO \\
\hline MTD & 600 & 90 & 0 & NO \\
\hline LTD & 400 & 90 & 0 & NO \\
\hline HTW & 800 & 90 & 0 & YES \\
\hline HTD10\% & 800 & 9 & 81 & NO \\
\hline
\end{tabular}

Once the test was completed, samples were cooled without interrupting the reducing gas flow. DC conductivity of the samples was then measured at room temperature. This final DC conductivity measurement accounts not only for the conductivity achieved during reduction, but also for the degradation due to nickel coarsening that took place under each conditions for the $20 \mathrm{~h}$ test.

Finally, the samples were observed in a Merlin FE-SEM (Carl Zeiss) in order to characterize at a qualitative level the morfology of the nickel microstructure generated during the 20h treatment. For this purpose samples where infiltrated with epoxy resin, ground and polished. Observations were made at low accelerating voltages in order to obtain contrast between the nickel and YSZ phases as described in (6) and using an EsB (Energy selective Backscattered) detector.

\section{Results and discussion:}

\section{Conductivity measurements:}

Results of the potentiostatic test for the first two hours are shown in figure 1. All samples part from an oxidized state with zero DC conductivity. After several minutes from the gas switch, $\mathrm{NiO}$ reduction is initiated and $\mathrm{DC}$ conductivity begins to rise in most samples. The reduction test at $400^{\circ} \mathrm{C}$ is the exception, where no DC conductivity was recorded during the whole duration of the test. All samples show a summit in DC conductivity (except for the one reduced at $400^{\circ} \mathrm{C}$ ) and then show a decay on conductivity over time, which can be attributed to Ni coarsening as a mechanism reducing the overall $\mathrm{Ni}$ phase connectivity. For samples reduced in a dry atmosphere and pure hydrogen the maximum conductivity was observed in the sample reduced at $800^{\circ} \mathrm{C}$, but falls rapidly to match the one recorded for the sample reduced at $600^{\circ} \mathrm{C}$. According to the theory of contiguity described in (7), this observation would evidence that as the same initial material was used, even though kinetics differ at this two temperatures, they rapidly tend to a similar degree of connectivity. While the samples reduced with pure hydrogen show a fast linear increase in DC conductivity, the sample reduced in the diluted reduction gas stream shows a much slower, and non linear increase in conductivity, which is only measurable after $1 \mathrm{~h}$ from the initial exposure to the reducing atmosphere. During this test, either several apparatus or connection failures occurred, causing periods in which no data was recorded, but without any manipulation, 
measurement was restored after several minutes. The conductivity of this sample rose to the highest recorded for the full set of samples during the test as can be observed in figure 2. This is consistent with water accelerating the $\mathrm{Ni}$ coarsening, as these conditions are probably the ones producing water at a slower rate, while the feed gas rate was kept the same as for other tests (i.e. same rate of gas to flush produced water out of the system).

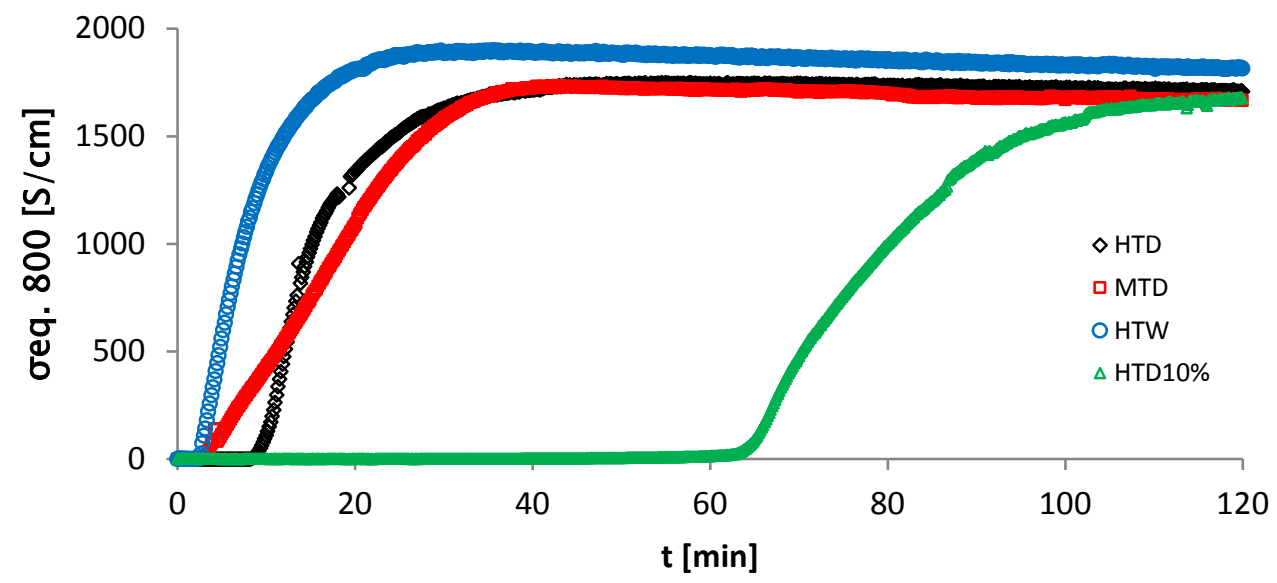

Figure 1. First 120 minutes of DC-conductivity test during reduction.

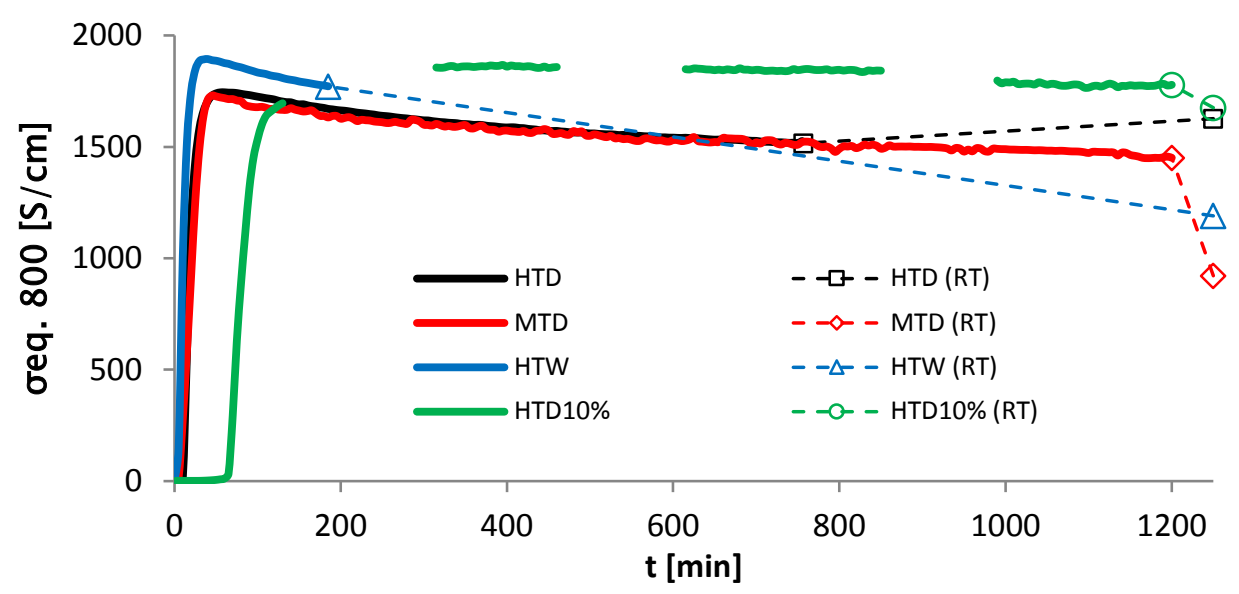

Figure 2. Full length of the DC conductivity test during reduction. Hollow squares at the right of the graph represent the equivalent conductivity at $800^{\circ} \mathrm{C}$ calculated from the measurements performed at room temperature after the test. Dotted lines connect these points with the last measured point in each test.

Results for the room temperature DC measurement after the 20h treatment are shown in table III. Figure 2 also shows a comparison between the last recorded value of DC conductivity measured in the reduction test and the value measured at room temperature (equivalently scaled to $800^{\circ} \mathrm{C}$ ). Surprisingly, the sample reduced at $600^{\circ} \mathrm{C}$ is the least conducting in the room temperature test. FESEM observation of the sample revealed small gaps between nickel particles, as for the sample reduced at $400^{\circ} \mathrm{C}$. A possible explanation of this fall of conductivity observed could be explained if some of these gaps could be closed when the sample is heated, as $\mathrm{Ni}$ has a higher thermal expansion coefficient than YSZ, enhancing the nickel phase connectivity at high temperatures. Apparently the temperature during these two tests was not sufficient to 
cause nickel adjacent particles to sinter during the test period. The sample reduced at $400^{\circ} \mathrm{C}$ still yields no DC conductivity, associated to a poor connectivity of metallic nickel particles, and also confirmed by SEM (see next section). The highest conductivity was achieved in the diluted feed sample, followed by the sample reduced at $800^{\circ} \mathrm{C}$ in a dry atmosphere and finally the largest degradation was seen for the sample reduced at $800^{\circ} \mathrm{C}$ in a humidified atmosphere. Most samples show lesser conductivities in the room temperature test, which could be explained by the loss of conductivity due to the Ni phase shrinkage on cooling. The reason for the sample reduced in the dry gas at $800^{\circ} \mathrm{C}$ yielding a higher conductivity than in the last measured point is not fully understood. The observed trend appears to point at higher conductivity values for the samples that were not exposed to a wet gas stream. Additionally this conductivity decay appears to be milder for samples with a lower water formation rate for the given flushing flow.

TABLE III. Final DC-conductivities for the samples performed at room temperature and comparison with the equivalent conductivity at room temperature (estimating a linear change in nickel conductivity) measured from the last recorded point in the reduction test before contact failure or the end of the test.

\begin{tabular}{|c|c|c|c|}
\hline $\begin{array}{c}\text { Sample } \\
\text { ID }\end{array}$ & $\begin{array}{c}\boldsymbol{\sigma} \mathbf{R T} \\
{\left[\mathbf{S c m}^{-1} \mathbf{]}\right.}\end{array}$ & $\begin{array}{c}\boldsymbol{\sigma R T} \text { from last recorded } \\
\text { point }\left[\mathbf{S c m}^{-1} \mathbf{]}\right.\end{array}$ & $\begin{array}{c}\text { t from last recorded } \\
\text { point [min] }\end{array}$ \\
\hline HTD & 9759 & 9096 & 757 \\
\hline MTD & 5529 & 8706 & 1200 \\
\hline LTD & 0 & 0 & - \\
\hline HTW & 7142 & 10626 & 185 \\
\hline HTD10\% & 10053 & 10668 & 1200 \\
\hline
\end{tabular}

Morphological observation:

Figure 3 shows the resulting microstructure after each test. Pores correspond to the black phase, YSZ is the darkest grey phase and nickel is the lighter grey phase. Pores can be seen in a bi-modal distribution: large pores were formed during the burnout of the pore former (corn starch) during the sintering step, while small pores (typical size under $1 \mu \mathrm{m}$ ) where formed during the reduction process.

Samples reduced at 600 and $400^{\circ} \mathrm{C}$ (figures 3.b and 3.c) show gaps surrounding nickel particles, and a lesser extent of nickel coarsening as compared to samples reduced at $800^{\circ} \mathrm{C}$. Moreover, some unreacted $\mathrm{NiO}$ can be observed in the sample reduced at $400^{\circ} \mathrm{C}$. Additionally both samples show internal porosity inside the nickel particles, though the effect is more evident in the sample reduced at $400^{\circ} \mathrm{C}$. Some pores are observable inside nickel particles in samples reduced at $800^{\circ} \mathrm{C}$ (figures 3.a, 3.d and 3.e). These observations could be explained by $\mathrm{NiO}$ reduction taking place inside the large particles while forming a pore channel inside the particles extending from the interface formed between the newly formed $\mathrm{Ni}$ crystallites and the $\mathrm{NiO}$ particle as reported in (8). Porous nickel particles are not stable surface-energy-wise, and they will tend to densify at a rate limited by nickel mobility, which could explain why this phenomenon is observed to a higher extent in the lower temperature experiments. However, it should be interesting to further study the formation and stability of these nanoporous nickel particles under different conditions. Although this purpose is out of the scope of the present work, those particles could present a huge range of potential application as catalysers. On the other 
hand, samples reduced with pure hydrogen at $800^{\circ} \mathrm{C}$ with and without added humidity show very similar microstructures, but the room temperature DC-conductivity measure points out that a higher loss of connectivity took place in the sample reduced with a humidified feed stream. In contrast with the gaps seen in the samples reduced at low temperature, no gap exist in most Ni-YSZ interfaces, At higher temperatures, YSZ becomes a good oxygen ion conductor and $\mathrm{NiO}$ reduction could take place through the NiO-YSZ interface (8-10). A lesser amount of nickel coarsening can be seen in the sample reduced in the diluted hydrogen flow when compared to the one reduced with the $800^{\circ} \mathrm{C}$ dry stream.
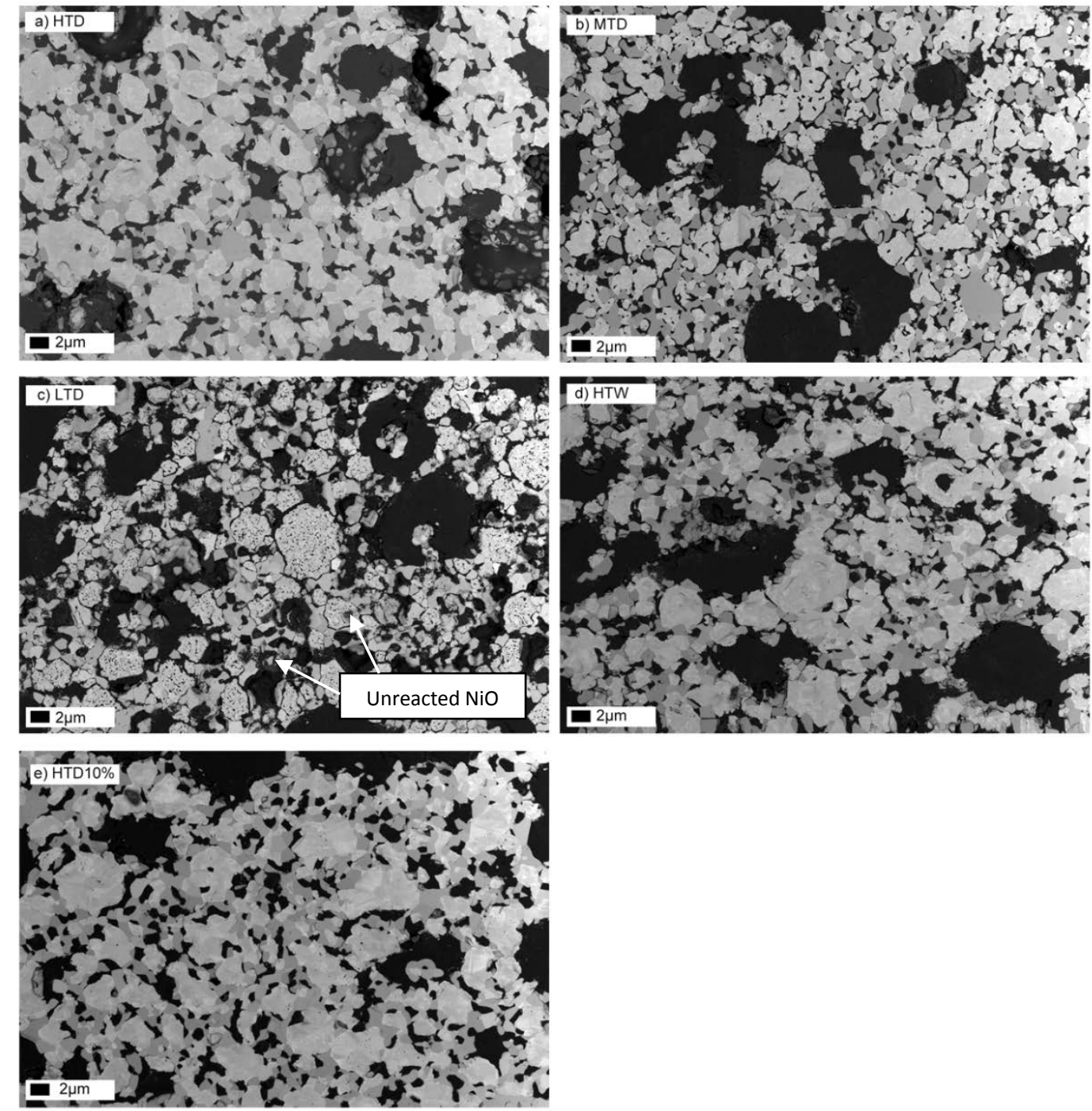

Figure 3. FESEM micrographs of the resulting microstructures after the 20h reduction test.

\section{Conclusions:}

A wide range of different microstructures can be formed from the same parting material. Even a non conducting material was formed during the lower temperature experiment. Not only the reduction mechanism and kinetics play a role in the final morphology, but also the nickel species mobility. The experiments described here 
support the hypothesis of nickel mobility being enhanced by added water presence. The mentioned species mobility also play a role in the $\mathrm{NiO}$ reduction kinetic behavior, but further experiments are required to elucidate the nature of this interaction. The difference observed between DC conductivities in the high and room temperature tests on the sample reduced at $600^{\circ} \mathrm{C}$ evidence that there is a difference in the effective microstructure between both temperatures, and for this reason no discussion was presented on the subject of TPB length. In order to fully understand those effects, electrochemical characterization under the working conditions seems to be the most reliable method and will be performed in a future work. Regarding exclusively DC conductivity at the end of the test, the best performance was obtained for the sample reduced in a diluted hydrogen atmosphere.

As a general conclusion, the reduction conditions of a Ni-YSZ cermet is crucial for its performance as a SOFC anode, especially during the first hours of operation, as their properties can drastically change during this period. For this reason, it is hard to validate most of the literature data for comparison purposes. Preconditioning of Ni-YSZ anodes until reaching an steady-state microstructure would be desirable.

\section{Acknowledgments:}

Authors would like to thak the project MAT2012-30763, financed by the Spanish Government (Ministerio de Economía y Competitividad) and the Feder program of the European Union. Authors would also acknowledge the use of Servicio General de Apoyo a la Investigación-SAI, Universidad de Zaragoza.

\section{References:}

1. $\quad$ H. Monzón, et al., Int. J. Hydrogen Energy, 39,10(2014)

2. J.T. Richardson, R. Scates, and M.V. Twigg, Applied Catalysis A: General, 246,1(2003)

3. $\quad$ A.F. Benton and P.H. Emmett, J. Am. Chem. Soc., 46,12(1924)

4. J. Sehested, et al., J. Catal., 223,2(2004)

5. $\quad$ M.H. Pihlatie, et al., Solid State Ionics, 189,1(2011)

6. $\quad$ K. Thydén, Y.L. Liu, and J.B. Bilde-Sørensen, Solid State Ionics, 178,39-40(2008)

7. D. Simwonis, F. Tietz, and D. Stöver, Solid State Ionics, 132,3-4(2000)

8. Q. Jeangros, et al., Acta Mater., 58,14(2010)

9. $\quad$ E.C. Dickey, et al., Microsc. Microanal., 3,05(1997)

10. M.A. Laguna-Bercero, et al., J. Eur. Ceram. Soc., 25,8(2005) 